\title{
Distribution, identification and genetic diversity among bamboo species: a phenomic approach
}

\begin{abstract}
Bamboo is grown irrespective of latitude, altitude and climatic factors on the planet earth. Despite its immense importance in human life from cradle to coffin, the study of distribution of bamboo and their identification is still very limited. On this backdrop, an extensive survey on entire bamboo resources was conducted at South 24 Parganas $\left(22^{\circ} 20^{\prime} \mathrm{N}\right.$ to $22^{\circ} 06^{\prime} \mathrm{N}, 88^{\circ} 20^{\prime} \mathrm{E}$ to $\left.88^{\circ} 60^{\prime} \mathrm{E}\right)$ district of West Bengal, India to document the distribution pattern of bamboo species followed by their identification. For selection of study area (blocks, gram panchayats and villages), probabilistic random sampling was used whereas non-probabilistic snow ball sampling was adopted for selection of respondents. Based on vegetative characters, species identification was done by Botanical Survey of India, Kolkata. Twenty-eight key photometric characteristics were recorded and the species diversity was analyzed. A total of 600 accessions were sampled which comprises 43 different variants. Of which Bambusa balcooa was the most predominant species in South 24 Parganas followed by Bambusa vulgaris. These two species are grown widely because of their suitability in construction of houses, bridges and also in agricultural activities. It is evident that the usability of the species was the chief reason of the diversity of bamboo species in the study area. Furthermore, the variation in the species may also be caused by the natural stress condition i.e. the variation in the level of salinity of the study area. The cluster analysis grouped the genotypes into ten different clusters.
\end{abstract}

Keywords: bamboo, distribution, diversity, identification, utility
Volume 7 Issue 2 - 2017

\author{
Lucina Yeasmin,' Md Nasim Ali, ${ }^{2}$ Syandan \\ Sinha Ray,' Pushpa Kumari ${ }^{3}$ \\ IIRDM Faculty Centre, School of Agriculture and Rural \\ Development, Vivekananda University, India \\ ${ }^{2}$ Department of Agricultural Biotechnology, Bidhan Chandra \\ Krishi Viswavidyalaya, India \\ ${ }^{3}$ Scientist C, AJC Bose Indian Botanic Garden, Botanical Survey \\ of India
}

Correspondence: Md Nasim Ali, Department of Agricultural Biotechnology, Bidhan Chandra Krishi Viswavidyalaya, Mohanpur, Nadia-74I252, India, Email nasimali2007@gmail.com

Received: May II, 2017| Published: June 08, 2017

\section{Introduction}

The fastest growing perennial, evergreen, arbores cent bamboo plant is a member of the grass family Poaceae and constitutes a single subfamily Bambusoideae. ${ }^{1}$ The distribution of bamboos on planet earth extends from $51^{\circ} \mathrm{N}$ in Japan to $47^{\circ} \mathrm{S}$ in South Argentina. The bamboo can grow in an altitudinal range which extends from just above the mean sea level up to $4,000 \mathrm{~m} .{ }^{2}$ Bamboo can also thrive in hot, humid rainforests to cold resilient forests. It can tolerate extreme temperature of about $-20^{\circ} \mathrm{C}$ as well as excessive annual precipitation ranging from 32 to 50 inch. ${ }^{3}$ About 14 million hectares of the earth surface is covered by bamboos with 80 percent in Asia. ${ }^{4} \mathrm{~A}$ total number of 1,400 bamboo species are distributed worldwide. The Bambusa tribe includes about 1,290 species worldwide and constitutes three major groups. ${ }^{5}$ The major species richness is found in Asia-pacific followed by South America, whereas the least number of species is found in Africa and India is the second richest country in bamboo genetic resources next to China. ${ }^{6}$ Several studies have been conducted to record the distribution of bamboo species in India. The report says that the number of species varies from $102^{7}$ to $136 .{ }^{8}$ As opined by many scientists, the distribution of bamboo is greatly influenced by agroclimatic zones, ${ }^{9}$ human interventions ${ }^{10}$ and climatic factors. ${ }^{11}$ Overexploitation and genetic erosion of bamboo species have made it necessary not only for the collection and conservation of its germplasm ${ }^{12,13}$ but also to classify and characterize them. ${ }^{14-16}$ Characterization of germplasm is an important link between the conservation and utilization of germplasm. ${ }^{17,18}$ To maintain the germplasm and conservation of biodiversity, the investigation of bamboo resources and even study of their local distribution is indispensable which is recorded to be limited till date in bamboo. The study of bamboo distribution in West Bengal is limited to northern part of the state. ${ }^{3}$ The present study was conductedto estimate the genetic diversity among the bamboo species distributed in the district of South 24 Parganas after proper documentation and, identification of them.

\section{Materials and methods}

\section{Survey, sample collection and identification}

An extensive survey on entire non-forest bamboo resource was conducted in South 24 Parganas $\left(22^{\circ} 20^{\prime} \mathrm{N}\right.$ to $22^{\circ} 06^{\prime} \mathrm{N}, 88^{\circ} 20^{\prime} \mathrm{E}$ to $88^{\circ} 60^{\prime} \mathrm{E}$ ) of West Bengal, India. Multistage sampling technique was adopted for the completion of survey. For selection of blocks, gram panchayats and villages probabilistic random sampling was used. For selection of respondents non probabilistic snow ball sampling was adopted. To conduct the survey work an interview schedule was constructed in local language which includes 3 parts: The first part consists of basic questions regarding the background of respondents; the Second part consists of location and local name of the species along with the land holding; the third part is regarding the usability and economic benefit of the bamboo species. Hherbarium samples were sent to Botanical Survey of India (BSI), Kolkata, for identification and preservation. The accession number for all the entries was collected (Table 1) from BSI.

\section{Morphological characterization}

A total of 600 accessions were sampled which comprises 43 different variants. Data on 28 key photometric characteristics (6 quantitative and 22 qualitative characters) as suggested by Bhattacharya et al., ${ }^{21}$ were recorded of 43 accessions and also of eight standard samples (all the 8 species found in the district during survey) maintained by BSI. Two accession (Acc. No. 68501 and 68503) identified as Bambusa oliveriana for which no standard sample was available. 


\section{Statistical analysis}

Three different culms were selected randomly from each clump or bamboo stand. Mean values from three independent replications were calculated for each quantitative characters. The qualitative data on culm and Culm sheath descriptors were then converted to a scale (showed as supplementary Table 4). Analysis of variance (ANOVA) and descriptive statistics were carried out for replicated quantitative data of culm and culm sheath using SPSS ver 16.0. Genetic diversity as depicted in the form of a dendrogram was constructed utilizing both qualitative traits and mean of quantitative traits using NTsys pc V 2.2. ${ }^{19}$ Data on 28 descriptors were transformed into $(0,1)$ after selecting mean as subtract option and standard deviation as divide option. Euclidean dissimilarity coefficient was opted for the construction of the dissimilarity matrix among the genotypes. The Unweighted Pair- Group Method of Arithmetic average (UPGMA). ${ }^{20}$ As clustering method and WARN in case of ties were used and dendrogram (Figure 1) was constructed.

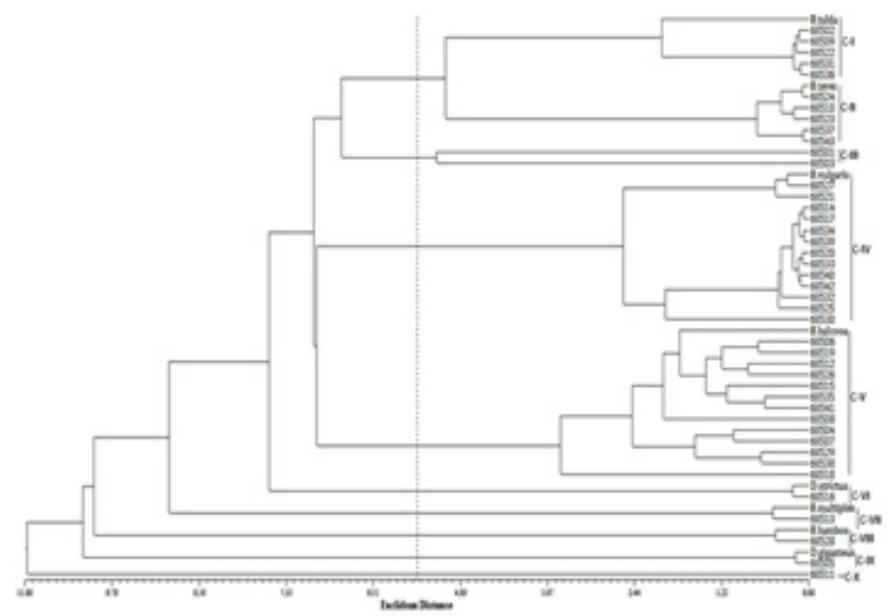

Figure I Dendrogram showing clustering pattern among 51 bamboo genotypes based on 28 Morphometric characteristics.

\section{Results}

Distribution, identification and utility of the bamboo species

A total of 600 entries were sampled from field which comprises
43 different variants for which accession was given by BSI, Kolkata (Table 1). All the 43 accessions were identified and divided into 9 different species (Figure 2) of bamboo under 2 genera after authentication from bamboo taxonomist of Botanical Survey of India (BSI). The two genera of bamboo, viz. Dendrocalamus and Bambusa were found in the study area. Bambusa constitutes 7 species and 2 species were recorded under Dendrocalamus. Bambusa balcooa was the most predominant species in the study area followed by Bambusa vulgaris (Figure 2). Besides, B. tulda, B. teres, B. oliveriana, B. multiplex, B. bamboos, D. strictus and D. giganteus were also found. It was evident from the present study that in different regions of the study area local names of the species was varied widely. A particular species of the bamboo was called with different local name e.g., B. balcooa were locally called as 'Gente (68504)','Dhuli (68506)', Bhalki (68508), Kechi (68518) and Bhalko (68538). On the contrary, the accession having same common name were identified as different species e.g., Acc. No 68503 and 68513 having common name 'Chhip bamboo' were respectively identified as $B$. oliveriana and $B$. multiplex. The Gente (68516) was even identified as different genus as Dendrocalamus strictus. From the survey report it was found that 520 acres of area was cultivated under bamboo cultivation in non- forest region of South $24 \mathrm{Pgs}$ district by the farmers. Species dependent multipurpose usability of different bamboos were established from the present study. Bambusa balcooa is a mechanically strong bamboo and it is mainly utilized for construction of houses and bridges. House and bridge construction, agriculture and allied activities consume $87 \%$ of the total bamboo production (Figure 3 ).

\section{Photometric characteristics}

Analysis of variance showed statistically significant variation among the accessions with respect to all the quantitative characters under study (Table 2). Huge variation was recorded for all the characters $(29-41 \%)$. The variation is mainly due to the age difference among the accessions. The mean of the characters is given in (Table 3 ). Maximum and minimum culm height was recorded for $D$. gianteus $(30 \mathrm{~m})$ and B. multiplex $(2.8 \mathrm{~m})$ with SE $( \pm \mathrm{m}) 0.45$. The length of the $5^{\text {th }}$ internode was more than $20 \mathrm{~cm}$ for most of the species except $B$. vulgaris. The ration of culm diameter to cavity diameter was least in case of $B$. teres $(0.04-0.05 \mathrm{~cm})$ whereas it was ten times higher in B. tulda and B. vulgaris $(>0.40)$. B. balcooa recorded lesser ratio of length and breadth of culm sheath. No such variation among the species was observed with respect to ration of length of culm sheath to length of blade.

Table I List of identified species based on phenology by botanical survey of India (BSI) along with BSI accession number and GPS location

\begin{tabular}{|c|c|c|c|c|}
\hline SI. No. & BSI accession & Local name & Scientific name & Location \\
\hline I. & 68501 & Muli & Bambusa oliveriana & Lat: 22015.014 'N/ Long: 88025.173” \\
\hline 2. & 68502 & Talda & Bambusa tulda & Lat: 22015.04'N/ Long: 88025.19”E \\
\hline 3. & 68503 & Chhip & Bambusa oliveriana & Lat: 22015.04'N/ Long: 88025.19"E \\
\hline 4. & 68504 & Gente & Bambusa balcooa & Lat: $22015.20^{\prime} \mathrm{N} /$ Long: 88025.16'E \\
\hline 5. & 68505 & Hom & Dendrocalamus giganteus & Lat: $22015.26^{\prime} \mathrm{N} /$ Long: $88025.18^{\prime} \mathrm{E}$ \\
\hline 6. & 68506 & Dhuli & Bambusa balcooa & Lat: 22015.3 'N/ Long: 88025. I 3'E \\
\hline 7. & 68507 & Gente & Bambusa balcooa & Lat: 22015.307 N/ Long: 88025.11 9’E \\
\hline 8. & 68508 & Bhalki & Bambusa balcooa & Lat: $22015.296^{\prime} \mathrm{N} /$ Long: $88025.176^{\prime} \mathrm{E}$ \\
\hline 9. & 68509 & Kulor & Bambusa tulda & Lat: $22015.056^{\prime} \mathrm{N} /$ Long: $88025.078^{\prime} \mathrm{E}$ \\
\hline 10. & 68510 & Java & Bambusa teres & Lat: $22015.635^{\prime} \mathrm{N} /$ Long: $88025.332{ }^{\prime} \mathrm{E}$ \\
\hline $\mathrm{II}$. & 68511 & Talda & Bambusa tulda & Lat: 22015.882 'N/ Long: 88025.372 'E \\
\hline
\end{tabular}


Table Continued..

\begin{tabular}{|c|c|c|c|c|}
\hline SI. No. & BSI accession & Local name & Scientific name & Location \\
\hline 12. & 68512 & Gente & Bambusa balcooa & Lat: 22015.587 'N/ Long: 88024.327’E \\
\hline 13. & 68513 & Chhip & Bambusa multiplex & Lat: 220 I2.59'N/ Long: 880 I5.346’E \\
\hline 14. & 68514 & Bashini & Bambusa vulgaris & Lat: $22011.98 \mathrm{I}$ 'N/ Long: $88015.309^{\prime} \mathrm{E}$ \\
\hline 15. & 68515 & Bhalki & Bambusa balcooa & Lat: $22013.18^{\prime} \mathrm{N} /$ Long: $88014.263^{\prime} \mathrm{E}$ \\
\hline 16. & 68516 & Gente & Dendrocalamus strictus & Lat: 2201 I.995'N/ Long: $88012.918^{\prime} E$ \\
\hline 17. & 68517 & Bashini & Bambusa vulgaris & Lat: 22013.503 'N/ Long: $88016.683^{\prime} \mathrm{E}$ \\
\hline 18. & 68518 & Kechi Bhalko & Bambusa balcooa & Lat: 22036.506'N/ Long: 88035.487’E \\
\hline 19. & 68519 & Bhalko & Bambusa balcooa & Lat: 22036.395 'N/ Long: 88034.260'E \\
\hline 20. & 68520 & Baria & Bambusa vulgaris & Lat:: $21^{\circ} 47.41 I^{\prime} \mathrm{N} /$ Long: $88^{\circ} 21.617^{\prime} \mathrm{E}$ \\
\hline 21. & 68521 & Bhalki & Bambusa vulgaris & Lat:: $21^{\circ} 47.4^{\prime} \mathrm{N} /$ Long: $88^{\circ} 21.630^{\prime} \mathrm{E}$ \\
\hline 22. & 68522 & Talda & Bambusa tulda & Lat:: $21^{\circ} 47.378^{\prime} \mathrm{N} /$ Long: $88^{\circ} 21.730^{\prime} \mathrm{E}$ \\
\hline 23. & 68523 & Kaba & Bambusa teres & Lat:: $21^{\circ} 47.319^{\prime} \mathrm{N} /$ Long: $88^{\circ} 21.943^{\prime} \mathrm{E}$ \\
\hline 24. & 68524 & Kal dhemni & Bambusa teres & Lat:: $21^{\circ} 47.257^{\prime} \mathrm{N} /$ Long: $88^{\circ} 21.335^{\prime} \mathrm{E}$ \\
\hline 25. & 68525 & Baria & Bambusa vulgaris & Lat:: $21^{\circ} 47.445^{\prime} \mathrm{N} /$ Long: $88^{\circ} 21.197^{\prime} \mathrm{E}$ \\
\hline 26. & 68526 & Gente & Bambusa balcooa & Lat:: $21^{\circ} 47.278^{\prime} \mathrm{N} /$ Long: $88^{\circ} 21.537^{\prime} \mathrm{E}$ \\
\hline 27. & 68527 & Guri Bhalki & Bambusa vulgaris & Lat:: $21^{\circ} 47.278^{\prime} \mathrm{N} /$ Long: $88^{\circ} 21.537^{\prime} \mathrm{E}$ \\
\hline 28. & 68528 & Kanta & Bambusa bambos & Lat:: $21^{\circ} 37.270^{\prime} \mathrm{N} /$ Long: $88^{\circ} 18.337^{\prime} \mathrm{E}$ \\
\hline 29. & 68529 & Gente & B. balcooa & Lat: 22007.790 'N/ Long: 88028.309' E \\
\hline 30. & 68530 & Lata falko & B. Vulgaris & Lat: 22007.7I 9'N/ Long: 88028.566' E \\
\hline 31. & 68531 & Talda & B. tulda & Lat: 22008.244'N/ Long: 88026.073' E \\
\hline 32. & 68532 & Bauni & B.Vulgaris & Lat:22008.253'N/ Long: 88026.055' E \\
\hline 33. & 68533 & Kali Bhalki & B.Vulgaris & Lat:22045.666'N/ Long:880I5.362' E \\
\hline 34. & 68534 & Bere & B. Vulgaris & Lat:22045.660'N/ Long:880I5.376' E \\
\hline 35. & 68535 & Gente & B. balcooa & Lat: 22045.659' N/ Long:880 I5.37I' E \\
\hline 36. & 68536 & Talda & B. tulda & Lat: 22045.599' N/ Long: 880 I5.507' E \\
\hline 37. & 68537 & Jawa & B. teres & Lat: 2201 I.87' N/ Long: 88042.937' E \\
\hline 38. & 68538 & Bhalko & B. balcooa & Lat: 220II.882' N/ Long: 88042.937' E \\
\hline 39. & 68539 & Bashini & B.Vulgaris & Lat: 22016.4 I I' N/ Long: 8804I.770' E \\
\hline 40. & 68540 & Bashini & B. Vulgaris & Lat: 22016.342' N/ Long: 8804I.782' E \\
\hline 41. & $6854 \mid$ & Bhalko & B. balcooa & Lat:22045.659' N/ Long: 880I5.376'E \\
\hline 42. & 68542 & Bashini & B. Vulgaris & Lat: 22045.659' N/ Long: 880 I5.376' \\
\hline 43. & 68543 & Jawa & B. teres & Lat: 22018.232' N/ Long: 88035.056'E \\
\hline
\end{tabular}

Table 2 Comparison of qualitative morphological parameters of bamboo genotypes growing under study area along with standard sample growing in BSI: Scale of characters are as follows: I. Internode Bending (absent: 0, present: I), 2. Colour (Dark green: 0, Bright green: I, Glossy green: 2, Pale green: 3, Grey green: 4, Yellow green: 5), 3. Swollen Node (absent: 0, present: I), 4. Nodal Ring (absent: 0, whitish: I, grayish: 2), 5. Nodal sheath Scar 6. Hairs at Nodal Ring, 7. Piercing Culm Sheath, 8. Curved Lower Nodal Branches (absent: 0, present: I), 9. Modification of Branches (none: 0, thorns: I, spines: 2), I0. Striation on Culm (absent: 0, present: I), I . Ciliate Margin, I2. Pubescentadaxial Side, I3. Pubescentabaxial Side, I4. Hair Colour (none: 0, golden brown: I, brown: 2, dark brown : 3, black: 4), 15. Number of Hairs (absent: 0, profuse: I, scanty: 2), I6. Shape of Blade (triangular: 0, lanceolate: I, acuminate: 2, ovate: 3), 17. Blade Reflexed, 18. Hairy Margin of Blade, 19.Auricle, 20. Auricle Continuous with Blade, 21 . Bristles on Auricle, 22. Auricle Fringed (absent: 0, Present: I)

\begin{tabular}{llllllllllllllllllllllllllll}
\hline Accessions & I & $\mathbf{2}$ & $\mathbf{3}$ & $\mathbf{4}$ & $\mathbf{5}$ & $\mathbf{6}$ & $\mathbf{7}$ & $\mathbf{8}$ & $\mathbf{9}$ & $\mathbf{1 0}$ & $\mathbf{1 1}$ & $\mathbf{1 2}$ & $\mathbf{1 3}$ & $\mathbf{1 4}$ & $\mathbf{1 5}$ & $\mathbf{1 6}$ & $\mathbf{1 7}$ & $\mathbf{1 8}$ & $\mathbf{1 9}$ & $\mathbf{2 0}$ & $\mathbf{2 1}$ & $\mathbf{2 2}$ \\
\hline Bambusa tulda & 0 & 0 & 0 & $\mathrm{I}$ & 0 & 0 & $\mathrm{I}$ & 0 & 0 & 0 & 0 & 0 & $\mathrm{I}$ & 4 & $\mathrm{I}$ & 3 & 0 & 0 & $\mathrm{I}$ & $\mathrm{I}$ & $\mathrm{I}$ & $\mathrm{I}$ & \\
68502 & 0 & 0 & 0 & $\mathrm{I}$ & $\mathrm{I}$ & 0 & $\mathrm{I}$ & 0 & 0 & 0 & 0 & 0 & $\mathrm{I}$ & 4 & $\mathrm{I}$ & 3 & 0 & 0 & $\mathrm{I}$ & $\mathrm{I}$ & $\mathrm{I}$ & $\mathrm{I}$ & \\
68509 & 0 & 0 & 0 & $\mathrm{I}$ & $\mathrm{I}$ & 0 & $\mathrm{I}$ & 0 & 0 & 0 & 0 & 0 & $\mathrm{I}$ & 4 & $\mathrm{I}$ & 3 & 0 & 0 & $\mathrm{I}$ & $\mathrm{I}$ & $\mathrm{I}$ & $\mathrm{I}$ & \\
$685 \mathrm{II}$ & $\mathrm{I}$ & 0 & 0 & $\mathrm{I}$ & $\mathrm{I}$ & 0 & $\mathrm{I}$ & 0 & 0 & $\mathrm{I}$ & 0 & 0 & $\mathrm{I}$ & 4 & $\mathrm{I}$ & 3 & 0 & 0 & $\mathrm{I}$ & $\mathrm{I}$ & $\mathrm{I}$ & $\mathrm{I}$ & \\
68522 & 0 & 0 & 0 & $\mathrm{I}$ & $\mathrm{I}$ & 0 & $\mathrm{I}$ & 0 & 0 & 0 & 0 & 0 & $\mathrm{I}$ & 4 & $\mathrm{I}$ & 3 & 0 & 0 & $\mathrm{I}$ & $\mathrm{I}$ & $\mathrm{I}$ & $\mathrm{I}$ &
\end{tabular}


Table Continued.

\begin{tabular}{|c|c|c|c|c|c|c|c|c|c|c|c|c|c|c|c|c|c|c|c|c|c|c|}
\hline 68531 & 0 & 0 & 0 & I & I & 0 & 1 & 0 & 0 & 0 & 0 & 0 & I & 4 & I & 3 & 0 & 0 & I & I & I & 1 \\
\hline Accessions & I & 2 & 3 & 4 & 5 & 6 & 7 & 8 & 9 & 10 & I I & 12 & 13 & 14 & 15 & 16 & 17 & 18 & 19 & 20 & 21 & 22 \\
\hline 68536 & 0 & 0 & 0 & I & I & 0 & I & 0 & 0 & 0 & 0 & 0 & I & 4 & I & 3 & 0 & 0 & I & I & I & I \\
\hline B. vulgaris & 0 & I & 0 & 0 & 0 & I & 0 & 0 & 0 & 0 & I & 0 & I & 3 & I & 0 & 0 & 0 & I & 0 & I & 0 \\
\hline 68514 & 0 & I & 0 & 0 & 0 & 1 & 0 & 0 & 0 & 0 & I & 0 & I & 3 & I & 0 & 0 & 0 & I & 0 & I & 0 \\
\hline 68517 & 0 & I & 0 & 0 & 0 & I & 0 & 0 & 0 & 0 & I & 0 & I & 3 & I & 0 & 0 & 0 & I & 0 & I & 0 \\
\hline 68520 & 0 & I & 0 & 0 & 0 & I & 0 & 0 & 0 & 0 & I & 0 & I & 3 & I & 0 & 0 & 0 & I & 0 & I & 0 \\
\hline 68521 & 0 & I & 0 & 0 & 0 & I & 0 & 0 & 0 & 0 & I & 0 & I & 3 & I & 0 & 0 & 0 & I & 0 & I & 0 \\
\hline 68525 & 0 & I & 0 & 0 & 0 & I & 0 & 0 & 0 & 0 & I & 0 & I & 3 & I & 0 & 0 & 0 & I & 0 & I & 0 \\
\hline 68527 & 0 & I & 0 & 0 & 0 & 1 & 0 & 0 & 0 & 0 & I & 0 & I & 3 & I & 0 & 0 & 0 & I & 0 & I & 0 \\
\hline 68530 & 0 & I & I & 0 & 0 & 1 & 0 & 0 & 0 & 0 & I & 0 & I & 3 & I & 0 & 0 & 0 & I & 0 & I & 0 \\
\hline 68532 & 0 & I & 0 & 0 & 0 & 1 & 0 & 0 & 0 & 0 & I & 0 & I & 3 & I & 0 & 0 & 0 & 1 & 0 & I & 0 \\
\hline 68533 & 0 & I & 0 & 0 & 0 & 1 & 0 & 0 & 0 & 0 & I & 0 & I & 3 & I & 0 & 0 & 0 & I & 0 & I & 0 \\
\hline 68534 & 0 & I & 0 & 0 & 0 & I & 0 & 0 & 0 & 0 & I & 0 & I & 3 & I & 0 & 0 & 0 & I & 0 & I & 0 \\
\hline 68539 & 0 & I & 0 & 0 & 0 & I & 0 & 0 & 0 & 0 & I & 0 & I & 3 & I & 0 & 0 & 0 & I & 0 & I & 0 \\
\hline 68540 & 0 & I & 0 & 0 & 0 & I & 0 & 0 & 0 & 0 & I & 0 & I & 3 & I & 0 & 0 & 0 & I & 0 & I & 0 \\
\hline 68542 & 0 & I & 0 & 0 & 0 & 1 & 0 & 0 & 0 & 0 & I & 0 & I & 3 & I & 0 & 0 & 0 & I & 0 & I & 0 \\
\hline B. balcooa & 0 & 0 & I & I & I & I & I & 0 & 2 & 0 & I & 0 & I & 4 & 2 & 2 & 0 & 0 & 0 & 0 & 0 & 0 \\
\hline 68504 & 0 & 0 & I & I & I & I & I & 0 & 2 & 0 & I & 0 & I & 4 & 2 & 2 & 0 & 0 & 0 & 0 & 0 & 0 \\
\hline 68506 & 0 & 0 & I & I & I & I & I & 0 & 2 & 0 & I & 0 & I & 4 & 2 & 2 & 0 & 0 & 0 & 0 & 0 & 0 \\
\hline 68507 & 0 & 0 & I & I & I & I & I & 0 & 2 & 0 & I & 0 & I & 4 & 2 & 2 & 0 & 0 & 0 & 0 & 0 & 0 \\
\hline 68508 & 0 & 0 & I & I & I & I & I & 0 & 2 & 0 & I & 0 & I & 4 & 2 & 2 & 0 & 0 & 0 & 0 & 0 & 0 \\
\hline 68512 & 0 & 0 & I & I & I & 1 & 1 & 0 & 2 & 0 & I & 0 & I & 4 & 2 & 2 & 0 & 0 & 0 & 0 & 0 & 0 \\
\hline 68515 & 0 & 0 & 1 & I & I & 1 & 1 & 0 & 2 & 0 & I & 0 & I & 4 & 2 & 2 & 0 & 0 & 0 & 0 & 0 & 0 \\
\hline 68518 & 0 & 0 & I & I & I & 2 & 1 & 0 & 0 & 0 & I & 0 & I & 4 & 2 & 2 & 0 & 0 & 0 & 0 & 0 & 0 \\
\hline 68519 & 0 & 0 & 1 & I & I & 1 & 1 & 0 & 2 & 0 & 1 & 0 & I & 4 & 2 & 2 & 0 & 0 & 0 & 0 & 0 & 0 \\
\hline 68526 & 0 & 0 & I & I & I & I & I & 0 & 2 & 0 & I & 0 & I & 4 & 2 & 2 & 0 & 0 & 0 & 0 & 0 & 0 \\
\hline 68529 & 0 & 0 & 1 & I & I & I & I & 0 & 2 & 0 & 1 & 0 & I & 4 & 2 & 2 & 0 & 0 & 0 & 0 & 0 & 0 \\
\hline 68535 & 0 & 0 & I & I & I & I & I & 0 & 2 & 0 & I & 0 & I & 4 & 2 & 2 & 0 & 0 & 0 & 0 & 0 & 0 \\
\hline 68538 & 0 & 0 & 1 & I & I & 1 & I & 0 & 2 & 0 & I & 0 & I & 4 & 2 & 2 & 0 & 0 & 0 & 0 & 0 & 0 \\
\hline 68541 & 0 & 0 & 1 & I & I & 1 & I & 0 & 2 & 0 & I & 0 & I & 4 & 2 & 2 & 0 & 0 & 0 & 0 & 0 & 0 \\
\hline D. giganteus & 0 & 4 & 0 & 3 & I & I & 0 & 0 & 0 & 0 & 0 & 0 & I & 2 & I & I & I & 0 & 0 & 0 & 0 & 0 \\
\hline 68505 & 0 & 4 & 0 & 3 & I & I & 0 & 0 & 0 & 0 & 0 & 0 & I & 2 & I & I & I & 0 & 0 & 0 & 0 & 0 \\
\hline B. multiplex & 0 & 3 & I & 0 & 0 & 0 & 0 & 0 & 0 & 0 & 0 & 0 & 0 & 0 & 0 & I & 0 & 0 & I & 0 & I & 0 \\
\hline 68513 & 0 & 3 & I & 0 & 0 & 0 & 0 & 0 & 0 & 0 & 0 & 0 & 0 & 0 & 0 & I & 0 & 0 & I & 0 & I & 0 \\
\hline B. teres & 0 & 0 & 1 & I & I & 1 & 1 & 0 & 0 & 0 & 0 & 0 & I & 2 & 2 & 2 & 0 & 0 & I & I & I & I \\
\hline 68510 & 0 & 0 & 1 & I & I & 1 & 1 & 0 & 0 & 0 & 0 & 0 & I & 2 & 2 & 2 & 0 & 0 & I & I & I & 1 \\
\hline 68523 & 0 & 0 & I & I & I & 1 & I & 0 & 0 & 0 & 0 & 0 & I & 2 & 2 & 2 & 0 & 0 & I & I & I & I \\
\hline 68524 & 0 & 0 & I & I & I & I & I & 0 & 0 & 0 & 0 & 0 & I & 2 & 2 & 2 & 0 & 0 & I & I & I & I \\
\hline 68537 & 0 & 0 & 1 & I & I & 1 & 1 & 0 & 0 & 0 & 0 & 0 & I & 2 & 2 & 2 & 0 & 0 & I & I & I & I \\
\hline 68543 & 0 & 0 & I & I & I & I & I & 0 & 0 & 0 & 0 & 0 & I & 2 & 2 & 2 & 0 & 0 & I & I & I & 1 \\
\hline B. bambos & 0 & 4 & I & 0 & 0 & 0 & 0 & I & I & 0 & I & I & 0 & 4 & 2 & 0 & 0 & 0 & I & I & I & I \\
\hline 68528 & 0 & 4 & 1 & 0 & 0 & 0 & 0 & I & I & 0 & I & I & 0 & 4 & 2 & 0 & 0 & 0 & I & I & I & 1 \\
\hline D. strictus & 0 & 4 & I & 0 & I & 0 & 0 & I & 0 & 0 & I & 0 & I & 2 & I & 2 & 0 & 0 & I & I & 0 & 0 \\
\hline 68516 & 0 & 4 & I & 0 & I & 0 & 0 & I & 0 & 0 & I & 0 & I & 2 & I & 2 & 0 & 0 & I & I & 0 & 0 \\
\hline 68501 & 1 & 3 & I & I & I & 1 & 0 & 0 & 0 & 0 & 0 & 0 & I & 2 & I & 2 & 0 & 0 & 1 & I & I & 1 \\
\hline 68503 & 0 & 3 & I & I & I & I & 0 & 0 & 0 & 0 & 0 & 0 & I & 2 & I & 2 & 0 & 0 & I & I & I & I \\
\hline
\end{tabular}


Table 3 Analysis of variance among accessions collected from study area for Culm height (CH), Culm Diameter (CD), length of $5^{\text {th }}$ internode (LI5), ratio of culm diameter to cavity diameter (CC), ratio of length and breadth of culm sheath at base (LB) and ratio of length of culm sheath to length of blade (LL)

\begin{tabular}{|c|c|c|c|c|c|c|c|}
\hline \multirow{2}{*}{ Sources of variation } & \multicolumn{7}{|c|}{ Mean sum of squares } \\
\hline & df & $\mathrm{CH}(\mathrm{m})$ & CD & $\mathrm{Cl} 5(\mathrm{~cm})$ & CC & LB & LL \\
\hline Between population & 50 & $91.653 * *$ & $|3| 9.6 \mid * *$ & $240.444 * *$ & $0.089 * *$ & $0.960 * *$ & $3.303^{* *}$ \\
\hline Error & 102 & 1.049 & 13.902 & 0.963 & 0.001 & 0.006 & 0.099 \\
\hline
\end{tabular}

Figure 2 Photographs of different Bamboo species encountered under study area. a. Bambusa balcooa, b. B. tulda, c. B. multiplex, d. D. giganteus, e. B. vulgaris, f. B. teres, g. B. oliveriana, h. D. strictus, i. B. bamboo.

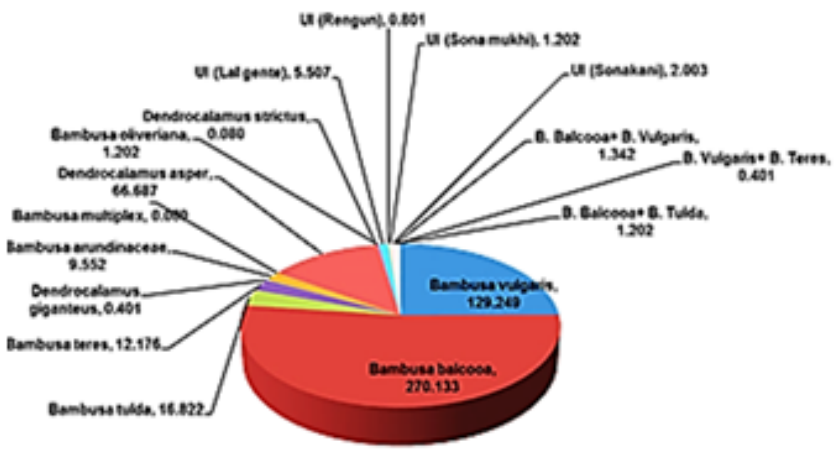

Figure 3 Area wise distribution of Bamboo species under study area. For unidentified species (UI) the local name is given within parenthesis. 
Table 4 Mean Performance of quantitative morphological parameters of bamboo genotypes growing under study area along with standard samples growing in BSI

\begin{tabular}{|c|c|c|c|c|c|c|}
\hline Accessions & $\mathrm{CH}(\mathrm{m})$ & $C D(\mathrm{~mm})$ & CI5 (cm) & CC & LB & LL \\
\hline B. tulda & 18.33 & 56.33 & 38.4 & 0.43 & 1.08 & 3.67 \\
\hline 68502 & 19.17 & 56.66 & 39.2 & 0.42 & 1.09 & 3.64 \\
\hline 68509 & 19.4 & 57.33 & 40.17 & 0.43 & 1.1 & 3.67 \\
\hline 68511 & 18.33 & 55 & 38.7 & 0.42 & 1.06 & 3.64 \\
\hline 68522 & 19.5 & 58 & 39.53 & 0.45 & 1.09 & 3.67 \\
\hline 68531 & 18.67 & 55.33 & 38.63 & 0.43 & 1.05 & 3.64 \\
\hline 68536 & 18.83 & 56 & 38.9 & 0.45 & 1.05 & 3.66 \\
\hline B. vulgaris & 19.07 & 85.33 & 23.77 & 0.51 & 1.65 & 2.76 \\
\hline 68514 & 8.77 & 58.33 & 14.5 & 0.5 & 1.6 & 2.75 \\
\hline 68517 & 8.83 & 57.67 & 14.93 & 0.51 & 1.62 & 2.71 \\
\hline 68520 & 7.77 & 56 & 13.37 & 0.5 & 1.66 & 2.75 \\
\hline 68521 & 20.77 & 84.67 & 24.77 & 0.51 & 1.65 & 2.72 \\
\hline 68525 & 10.13 & 60.67 & 12.17 & 0.49 & 1.59 & 2.73 \\
\hline 68527 & 17.93 & 82.33 & 22.43 & 0.51 & 1.69 & 2.77 \\
\hline 68530 & 8.57 & 59.33 & 14.93 & 0.5 & 1.66 & 2.71 \\
\hline 68532 & 9.27 & 62 & 16 & 0.5 & 1.66 & 2.76 \\
\hline 68533 & 8.13 & 56.33 & 13.03 & 0.5 & 1.66 & 2.77 \\
\hline 68534 & 8.47 & 58.67 & 13.77 & 0.51 & 1.65 & 2.71 \\
\hline 68539 & 8.57 & 58.33 & 14.27 & 0.5 & 1.64 & 2.7 \\
\hline 68540 & 8.23 & 57.67 & 13.1 & 0.48 & 1.61 & 2.74 \\
\hline 68542 & 8.2 & 57.33 & 12.7 & 0.5 & 1.65 & 2.68 \\
\hline B. balcooa & 19.9 & 91 & 20.6 & 0.32 & 1.35 & 4.23 \\
\hline 68504 & 11.32 & 94.67 & 30.26 & 0.27 & 0.88 & 7.11 \\
\hline 68506 & 11.87 & 83 & 26.97 & 0.2 & 0.68 & 4.22 \\
\hline 68507 & 12.82 & 85.33 & 26.6 & 0.32 & 1.17 & 6.51 \\
\hline 68508 & 10.57 & 63 & 25.77 & 0.43 & 1.08 & 3.25 \\
\hline 68512 & 10.87 & 79 & 28.17 & 0.32 & 1.12 & 4.9 \\
\hline 68515 & 18.93 & 76.33 & 28.69 & 0.28 & 0.8 & 3.76 \\
\hline 68518 & 12.24 & 83.33 & 27.81 & 0.29 & 0.86 & 4.28 \\
\hline 68519 & 11.99 & 88 & 26.44 & 0.3 & 0.85 & 4.23 \\
\hline 68526 & 13.69 & 87.33 & 28.27 & 0.35 & 0.93 & 5.33 \\
\hline 68529 & 10.07 & 69.33 & 21.03 & 0.39 & 0.98 & 6.06 \\
\hline 68535 & 14.95 & 86 & 26.98 & 0.4 & 0.86 & 3.99 \\
\hline 68538 & 12.08 & 66.67 & 23.57 & 0.31 & 0.92 & 5.94 \\
\hline $6854 \mid$ & 16.63 & 92 & 30.77 & 0.4 & 0.94 & 4.1 \\
\hline D. giganteus & 30 & $|3|$ & 39.3 & 0.69 & 1.32 & 3.34 \\
\hline 68505 & 29.4 & 130 & 38.03 & 0.69 & 1.3 & 3.34 \\
\hline B. multiplex & 3.3 & 20.33 & 21.07 & 0.1 & 2.87 & 2.89 \\
\hline 68513 & 2.68 & 18.33 & 19.63 & 0.11 & 2.61 & 2.87 \\
\hline B. teres & 9.31 & 65.67 & 31.43 & 0.042 & 1.58 & 3.23 \\
\hline 68510 & 10.66 & 67.33 & 32.67 & 0.04 & 1.6 & 3.23 \\
\hline
\end{tabular}




\begin{tabular}{|c|c|c|c|c|c|c|}
\hline Accessions & $\mathrm{CH}(\mathrm{m})$ & $C D(\mathrm{~mm})$ & $\mathrm{Cl} 5(\mathrm{~cm})$ & $\mathrm{CC}$ & LB & LL \\
\hline 68523 & 11.78 & 67 & 33.03 & 0.05 & 1.58 & 3.24 \\
\hline 68524 & 9.43 & 64 & 31.47 & 0.05 & 1.6 & 3.22 \\
\hline 68537 & 13.53 & 73.33 & 33.5 & 0.04 & 1.58 & 3.22 \\
\hline 68543 & 13.84 & 73.67 & 33.1 & 0.04 & 1.6 & 3.24 \\
\hline B. bambos & 15.73 & 59 & 23.03 & 0.37 & 2.89 & 3.86 \\
\hline 68528 & 13.63 & 55.67 & 21.37 & 0.36 & 2.93 & 3.88 \\
\hline D. strictus & 15.07 & 49.33 & 37.4 & 0.61 & 1.38 & 3.85 \\
\hline 68516 & 14.1 & 49 & 36.27 & 0.6 & 1.4 & 3.85 \\
\hline 68501 & 14.27 & 39.33 & 35.27 & 0.52 & 2.67 & 2.37 \\
\hline 68503 & 7.97 & 37.33 & 35.03 & 0.53 & 2.67 & 2.35 \\
\hline Grand mean & 13.48 & 67.35 & 26.87 & 0.38 & 1.48 & 3.6 \\
\hline $\mathrm{SE}( \pm \mathrm{m})$ & 0.45 & 1.7 & 0.72 & 0.01 & 0.05 & 0.09 \\
\hline $\operatorname{LSD}(0.05)$ & 1.9 & 6.93 & 1.82 & 0.06 & 0.14 & 0.58 \\
\hline Range & $2.5-30.5$ & $18-135$ & II.8-40.5 & $0.04-0.70$ & $0.67-2.95$ & $2.31-7.80$ \\
\hline CV (\%) & 41.2 & 31.27 & 33.22 & 45.02 & 38.21 & 29.8 \\
\hline
\end{tabular}

\section{Genetic Diversity}

Dendrogram based on 28 phenometric characteristics was constructed to reveal the genetic diversity among the accessions. From the dendrogram, the genotypes were grouped into 10 different clusters. The distribution of the accessions into different clusters is shown in (Figure 4). It was evident that the most of accessions were clustered with their respective standard species with few exceptions. The accession 68511 identified as B. tulda was the member of a completely distant cluster (Cluster-X) from the others with maximum dissimilarity. The accessions 68501 and 68505 (morphologically identified as B. oliveriana,) for which no standard species were available, did not group with any other species. Most of population showed homogeneity (less dissimilarity coefficients) with their respective standard species except balcooa and tulda. The balcooa population was the most heterogeneous where all the accessions grouped in the same internode at dissimilarity coefficient more than 3.00. The pattern of diversity was in conformity with the morphological identification of the accession.

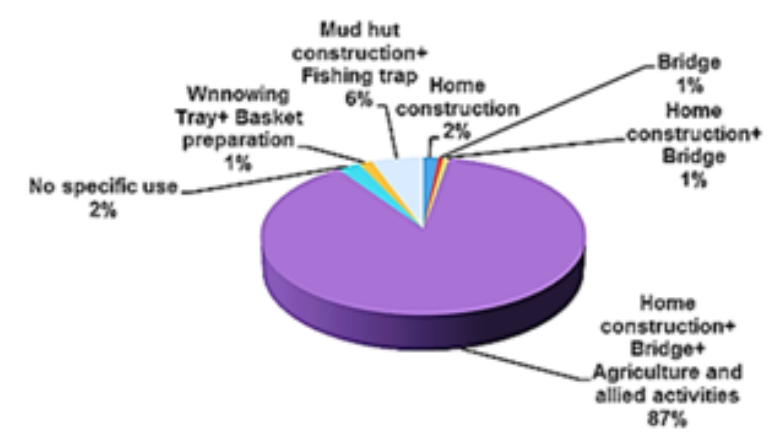

their identification, classification and proper documentation is still very limited in bamboo. The present study is aimed to documentation of the distribution of bamboo species in a district of West Bengal, India. The findings were in agreement with previous workers that the distribution of bamboo is greatly influenced by human intervention ${ }^{10}$ and climatic factors. ${ }^{11}$ This documentation and classification will facilitate the collection and conservation of germplasms ${ }^{14-16}$ at least in study area. In case of plant the identification keys are mostly based on floral characters. ${ }^{22}$ But, Incidence of flowering of woody bamboo is uncertain..$^{23,24}$ As reported earlier, the reproductive cycle of bamboo is too long from 3years to 120years. ${ }^{25}$ That is why the identification depending on reproductive structure is difficult. The present investigation classified and identified the bamboo collections based on vegetative characters (culm and cul-sheath characters) as suggested by many workers earlier. ${ }^{26-29}$ The genetic diversity vis-s-vis phylogenetic relationships among 15 bamboo species were evaluated by $\operatorname{Das}^{30}$ using morphological characters as done in this study. But the limitation of morphological characters which are influenced by the environmental factors was also evident in the study as reported by earlier reports. ${ }^{28}$ There are several instances of taxonomical discrepancies because of unstable vegetative characters. In the present investigation few accessions were not properly identified possibly because of the inability of morphological characters which are not adequate enough to separate the genus and also unsuitable for identification of closely related species., ${ }^{30,31}$ The molecular characterization is suggested for stable identification of the bamboo accessions in general and for closely related species in particular.

\section{Conclusion}

A total of 43 accessions collected from different locations of South 24 Parganas district of west Bengal, India. Almost all accessions were identified based on culm and culm sheath characters. The district was covered predominantly with Bambusa balcooa and Bambusa vulgaris species. Nine species under two genera i.e Bambusa and Dendrocalamus were found in the study area. The cluster analysis grouped all the accessions into 10 different clusters with the taxonomical discrepancy for an accession (68511) which stood alone in separate cluster though was identified as Bambusa balcooa.

\section{Discussion}

Bamboo is considered as "green gold" keeping in mind its economic importance and multiple end uses in human life, from cradle to coffin..$^{21}$ Despite of importance the study of distribution bamboo, 


\section{Acknowledgements}

The authors acknowledge DST, Govt. of India for providing fellowship to one of the author (INSPIRE fellow) and the authors are highly indebted to DST, Govt. of West Bengal, India for providing a fund to this project vide ref. grant (G. O.: 665 (Sanc.)/ST/P/S \& $\mathrm{T} / 1 \mathrm{G}-8 / 2011)$. The assistance received from Botanical Survey of India (BSI), Kolkata, India is also highly acknowledged.

\section{Conflict of interest}

Authors declare that there is no financial interest or conflict of interest.

\section{References}

1. Kigomo BN. Distribution, cultivation and research status of bamboo in Eastern Africa. KEFRIE col Ser Monogr. 1988;1:1-19.

2. Behari B. Status of Bamboo in India. Compilation of papers for preparation of national status report on forests and forestry in India. Survey and Utilization Division, Ministry of Environment and Forest; 2006. p. 109-120.

3. Goyal AK, Ghosh PK, Dubey PK, et al. Inventorying bamboo biodiversity of North Bengal: a case study. Int J Fund Appl Sci. 2012;1(1):5-8.

4. Tewari DN. A monograph on Bamboo. India: International Book Distributors; $1992.498 \mathrm{p}$.

5. Das M, Bhattacharya S, Singh P, et al. Bamboo taxonomy and diversity in the Era of molecular markers. Adv Bot Res. 2008;47:225-268.

6. Bystriakova N, Kapos V, Lysenko I, et al. Distribution and conservation status of forest bamboo biodiversity in the Asia-Pacific region. Biodiversity and Conservation. 2003;12(9):1833-1841.

7. Ohrnberger D. The bamboos of the World. Second impression. Amsterdam, Netherlands: Elsevier; 2002.

8. Sharma YML. Bamboos in the Asia Pacific Region. In: Lessard G, editors. Proceedings Workshop on bamboo research in Asia. Canada: International Development Research Centre; 1980. p. 99-120.

9. Varmah JC, Bahadur KN. Bamboo research in Asia, proceedings of a workshop in Singapore. In: Lessard G, et al. editors. India; 1998. p. 19-46.

10. Boontawee B. Status of bamboo research and development in Thailand In: proceedings of the International Bamboo workshop held in Cochin. India: Kerala Forest Research; 1998. p. 14-18.

11. Gamble JS. The Bambuseae of British India. India: Bengal Secretariat Press; 1896. p. 1-133.

12. IV Ramanuja Rao, Gnanaharan R, Cherla B Sastry. Genetic wealth of bamboos in India and their conservation strategies. Bamboos Current Research. In: Proceedings of International Bamboo workshop. India; 1988. 394 p.

13. Loh JP, Kiew R, Set O, et al. A study of genetic variation and relationship within the bamboo sub tribe Bambusinae using amplified fragment length polymorphism. Ann Bot. 2000;85(5):607-612.
14. Bahadur KN. Taxonomy of bamboos. Ind J For. 1979;2:222-241.

15. Soderstorm TR, Calderon CE. A commentary on bamboos (Poaceae: Bambusoideae). Biotropica. 1979;11(3):161-172.

16. Rao AN, Rao VR. Patterns of variation in bamboo. In: Williams JT, editors. Genetic enhancement of bamboos and rattan. India: International Network for Bamboo and Rattan; 1995.

17. Stapleton CM, Rao VR. Progress and Prospects in Genetic Diversity Studies on bamboo and its Conservation. Bamboo, People and the Environment. In the proceedings of $5^{\text {th }}$ International Bamboo Workshop and the IV International Bamboo Congress, Indonesia; 1995. p. 19-22.

18. Nayak S, Rout GR, Das P. Evaluation of the genetic variability in bamboo using RAPD markers. Plant Soil Environ. 2003;49(1):24-28.

19. Rohlf PJ. NTSYSpc. Numerical taxonomy and multivariate analysis system, version 2.2. Applied Biostatistics, USA; 1990

20. Sneath PH, Sokal RR. Numerical taxonomy. The principles and practice of numerical classification. W H Freeman and Company San Francisco. 1973;215(6):106-116.

21. Bhattacharya S, Ghosh JS, Das M, et al. Morphological and molecular characterization of Thamnocalamus spathiflorus subsp. spathiflorus at population level. Plant Systematics and Evolution. 2009;282(1):1-103.

22. Wu MCY. The Classification of bambuseae based on leaf anatomy. Botanical Bulletin of Academia Sinica. 1962;3:83-108.

23. Ramanayake SMSD, Meemaduma VN, Weerawardene TE. Genetic diversity and relationships between nine species of bamboo in Sri Lanka, using Random Amplified Polymorphic DNA. Plant Systemics and Evolution. 2007;269(1):55-61.

24. Mukherjee AK, Ratha S, Dhar S, et al. Genetic relationships among 22 taxa of bamboo revealed by ISSR and EST-based random primers. Biochemical Genetics. 2010;48(11-12):1015-1025.

25. Janzen DH. Why bamboos wait so long to flower. Ann Rev Ecolo Syst. 1976;7:347-391

26. Bennet SSR, Gaur RC. Thirty seven Bamboos growing in India. India: Forest Research Institute; 1990. 100 p.

27. Chatterjee RN, Raizada MB. Culm sheaths as an aid to identification of Bamboos. Indian Forester. 1963;89(11):744- 756.

28. Triplett J, Clark LG. Ambiguity and an American bamboo:The Chusquea culeou species complex. Bamboo Science and Culture:The Journal of the American Bamboo Society. 2003;17:21-27.

29. Bhattacharya S, Das M, Bar R, et al. Morphological and molecular characterization of Bambusa tulda with a note on flowering. Ann Bot. 2006;98(3):529-535.

30. Das M, Bhattacharya S, Basak J, et al. Phylogenetic relationships among the bamboo species as revealed by morphological characters and polymorphism analyses. Biologia Plantarum. 2007;51(4):667-672.

31. Sharma YML. Bamboos in the Asia Pacific Region. In: proceedings Workshop on Bamboo Research in Asia. Singapore, G Lessard, et al. editors. Canada: International Development Research Centre; 1980. p. 99-120. 